\title{
Classical Phase Space Revealed by Coherent Light
}

\author{
Tomoko Tanaka, ${ }^{1}$ Martina Hentschel,,${ }^{1,2}$ Takehiro Fukushima, ${ }^{1,3}$ and Takahisa Harayama ${ }^{1}$ \\ 1 Department of Nonlinear Science, ATR Wave Engineering Laboratories, \\ 2-2-2 Hikaridai, Seika-cho, Soraku-gun, Kyoto 619-0228, Japan \\ ${ }^{2}$ Institut für Theoretische Physik, Universität Regensburg, D-93040 Regensburg, Germany* \\ 3 Department of Communication Engineering, Okayama Prefectural University, Soja 719-1197, Japan
}

(Dated: November 8, 2018)

\begin{abstract}
We study the far field characteristics of oval-resonator laser diodes made of an AlGaAs/GaAs quantum well. The resonator shapes are various oval geometries, thereby probing chaotic and mixed classical dynamics. The far field pattern shows a pronounced fine structure that strongly depends on the cavity shape. Comparing the experimental data with ray-model simulations for a Fresnel billiard yields convincing agreement for all geometries and reveals the importance of the underlying classical phase space for the lasing characteristics.
\end{abstract}

PACS numbers: 42.25.Bs,05.45.Mt,42.55.Sa

Introduction - In the past two decades, quantum chaos has proven to be a successful concept in understanding, characterizing, and predicting the behavior of mesoscopic systems [1, 2]. Originally used to study the quantum analogue of classically chaotic hard-wall billiard model systems, it also explains the behavior of realistic systems of various shapes and character: The statistics of Coulomb-blockade peaks in quantum dots [2], the geometry dependence of the weak localization peak [3], or the level statistics of microwave billiards [4]. The studies on quantum chaos have mainly focused on the quest for universality from the view-point of statistical physics. Therefore, despite the evident importance of the system's underlying classical phase space for the behavior of the quantum or wave mechanical analogue (based on the analogy between Schrödinger and Helmholtz equation [1]), its specific structure cannot be reconstructed from the traces it leaves in typical observables like energy level or wave function statistics.

In this Letter we shall see that detailed information about the classical phase space can, however, be extracted from the far field radiation characteristics of ovalmicrocavity laser diodes. The observed far field pattern (FFP) depends very sensitively on the shape, that is, on the system's underlying classical phase space, and we convincingly support this idea by numerical simulations. It is interesting to note that information about the classical phase space is revealed by the coherent light emanating from the lasing microcavity.

The oval-billiard family is a well-known model system with interesting properties [5, 6, 7] in which each oval shape is characterized by a shape parameter $\delta \equiv 2 \delta^{\prime} / L$, cf. Fig. 1 (b). The curvature is, by construction [see Fig. 1(b) and, e.g., Ref. 7 for instructions], discontinuous. This was found to be crucial for understanding the phase-space evolution upon variation of $\delta$ [6]. Increasing $\delta$, the phase space changes from fully chaotic $(\delta=0$, stadium shape), via mixed ( $0<\delta<1$, various oval shapes) to integrable $(\delta=1$, disk). Figure 2 shows the phase
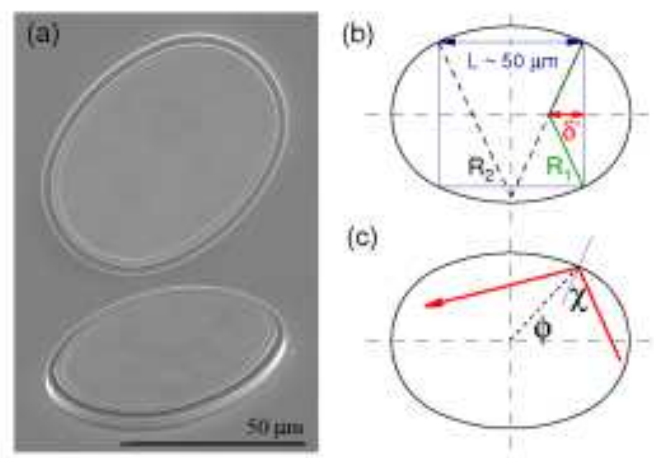

FIG. 1: (Color online) An oval microlaser with $\delta \equiv$ $2 \delta^{\prime} / L=0.45$. (a) Scanning Electron Micrograph, (b) geometry, and (c) phase-space coordinates.

space of the chaotic and integrable limit and the mixed phase spaces for $\delta \in[0.45,0.85]$ that consist of chaotic regions as well as regular islands [9]. The simultaneous experimental and theoretical investigation of a $\delta$-dependent mixed phase space has not, to the best of our knowledge, been done before and is at the core of the present study.

To understand the dynamics of the system, let us consider a light ray hitting the resonator boundary at polar angle $\phi$ under an angle of incidence $\chi$, cf. Fig:1(c), such that total internal reflection takes place $(|\sin \chi| \geq 1 / n)$. In the circular cavity $(\delta=1)$, the light ray will remain confined inside the cavity forever by means of conservation of angular momentum, and form whispering gallery mode (WGM) tori. To a certain extent, this scenario will remain valid even if $\delta$ is slightly decreased. The invariant WGM tori are then perturbed to Kolmogorov-ArnoldMoser tori, and the phase space becomes mixed. Note that for $\delta \geq 0.76$ the chaotic regions are disconnected (the chaotic sea is split into two disjoined regions) whereas for $\delta<0.76$ a singly connected chaotic layer penetrates the phase space, bypassing the embedded regular islands 

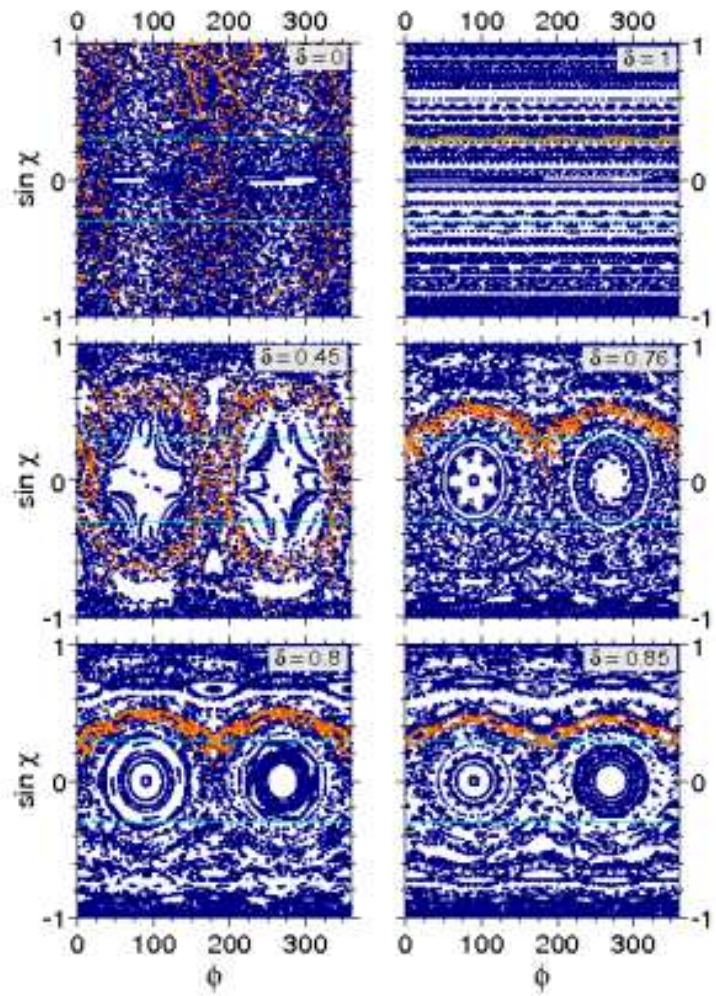

FIG. 2: (Color online) Poincaré surface of section (SOS) illustrating the classical phase space of hard-wall oval billiards. The critical lines $|\sin \chi|=1 / n$ are shown by light-blue lines. In the top raw, the limiting cases of a chaotic $(\delta=0$, stadium) and an integrable ( $\delta=1$, disk) phase space are shown; for $\delta=0.45,0.76,0.8$, and 0.85 the phase space is mixed. To further visualize the differences in the phase-space structure, the evolution of 50 rays with random initial conditions in the interval $0 \leq \phi \leq 0.1$ and $0.28 \leq \sin \chi \leq 0.32$ is followed for 25 reflections (superposed lighter points).

[6, 7], cf. Fig. 2, The smaller $\delta$ the more chaotic the cavity becomes, reaching ergodicity in the limit $\delta=0$.

The above mentioned properties of ray-dynamics in oval billiards imply in particular that when $\delta$ exceeds values of $\sim 0.76$, light rays are trapped in the disjoined chaotic regions that lie above the critical line $|\sin \chi|=1 / n$, unable to leave the cavity. Accordingly, the emitted light intensity is expected to decrease around $\delta \sim 0.76$ because the lasing modes will be strongly confined inside the cavity and leakage will be evanescent.

In order to elucidate if this property of classical phase space could be detected in the emitted light, we actually fabricated these oval-shaped semiconductor laser diodes for various values of $\delta$, one of which is shown in Fig. 1(a).

System - The resonators were made of metal organic vapor phase epitaxy grown graded index seperate confinement AlGaAs/GaAs single-quantum well wafer with $n=3.3$ the effective index of refraction. The typical system length is $L \sim 50 \mu \mathrm{m}$. Applying the reactive-ionbeam etching technique allows one to realize specific oval

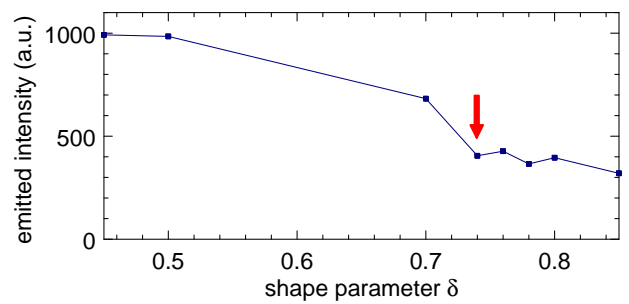

FIG. 3: Total emitted intensity of lasing microcavities vs. shape parameter $\delta$. Note the intensity drop (marked by arrow) around $\delta \sim 0.76$ caused by the appearance of a transversal barrier in phase.

cavities characterized by a shape parameter $\delta$ with extremely smooth and vertical boundaries, see Fig. 1(a); the surface roughness is less than 1/10 of the lasing wavelength $\lambda \sim 850 \mathrm{~nm}$. Lasing operation of these devices was achieved at room temperature by pumping with a pulsed injection current of $500 \mathrm{~ns}$ width at a $1 \mathrm{kHz}$ repetition rate.

Experiments - In Fig. 3 we plot the total (polar-angle integrated) far field output power, measured at an injection current of four times the threshold current, as function of the shape parameter $\delta$. The pronounced drop in the emitted light intensity around $\delta \lesssim 0.76$ (marked by arrow) is a direct consequence of the above-mentioned appearance of a transversal barrier in classical phase space and the change of the lasing modes from refractive modes into evanescent, WGM-type modes. This dip is also a clear sign that not individual modes, but the underlying classical phase space as a whole (representing a wealth of modes [8]) determines the behaviour of the system.

In the following, we will shift our focus from the total output power to the angular distribution of the emitted radiation in the far field, i.e., the FFPs. The experimental FFPs of oval lasing diodes with various $\delta$ are shown in Fig. 4 (black curves). FFPs were measured in a distance of $d=70 \mathrm{~mm}$ by scanning a photo-detector with a window of $w=11 \mathrm{~mm}$ width around the laser diode covering an angular range of $200^{\circ}$ in $\phi_{\mathrm{ff}}$ with a resolution $\Delta \phi_{\mathrm{ff}}=2 \arctan (w / 2 d) \sim 9^{\circ}$.

The red curves in Fig. 4 are the results of numerical, ray-model based simulations (explained in more detail below) that convincingly support the experimental results. A uniform background was substracted in all cases in order to exclude effects of spontaneous emission that is assumed to be isotropic; the (absolute) minimum of the experimental curves is set to zero by definition.

Model - The theoretical model used here is based on the ray picture. This is motivated by the large size parameter $\sim 2 \pi n L / \lambda \sim 1000$ of the experimental system; the ray model can be expected to yield reliable results in this regime. 9, 10] A large number (250000) of rays is started with unit amplitude and random initial conditions covering the whole phase space. The dynamics of 


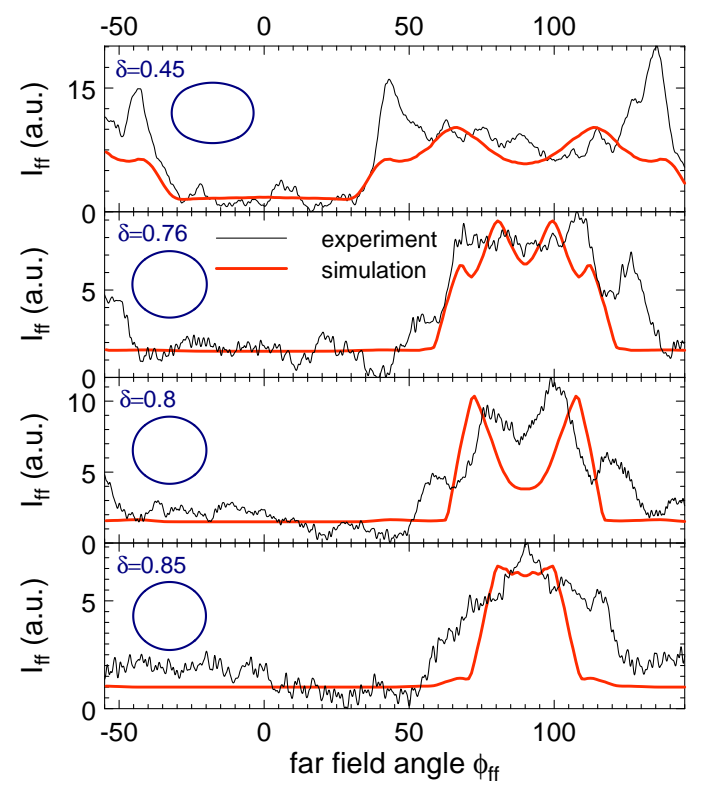

FIG. 4: (Color online) Experimentally observed FFPs (far field intensity $I_{\mathrm{ff}}$ vs. angle $\phi_{\mathrm{ff}}$, black curves; same injection currents as in Fig. 3 were used) for various shape parameters $\delta$ (see insets). The ray-model simulations are superimposed (red curves); all data was averaged over a $9^{\circ}$ window. The simulation data is offset by the average residual background of the experimental data, normalization is with respect to the peak heights. Apart from the side peaks, the agreement experiment-theory is convincing and the drastic changes in the FFP upon tiny changes in the shapes are nicely reflected in the simulation.

each ray is governed by Snell's and Fresnel's law [11] that complement the condition of specular reflection. The far field radiation characteristics is reconstructed from rays in the steady phase-space distribution [9, 12], an invariant object uniquely related to the underlying phase-space structure and characterized by an exponential decay of the internal light intensity vs. trajectory length. The initial transient regime where the internal intensity decays faster than exponentially due to the presence of, e.g., bouncing ball orbits, is discarded [9]. That the linear ray-model works so well for describing non-linear, lasing light is related to the large size-parameter and the fact that not a single, but a multitude of modes is lasing. [8]

Results - The observed FFPs exhibit a clear and prominent minimum-maximum structure that is remarkably well reproduced by the ray-model simulations for all $\delta$, cf. Fig. 4. Apart from the edges of the broad maxima where side peaks are sometimes missing, the simulated FFPs capture the decreasing width and fine structure of the central part, in particular its extreme shapesensitivity, in a semi-quantitative manner.

For all $\delta$ considered here, the preferred emission direction coincides with the shorter cavity axis $\left(\phi_{\mathrm{ff}} \sim 90^{\circ}\right)$. Note that this direction is shifted by $90^{\circ}$ in compari-

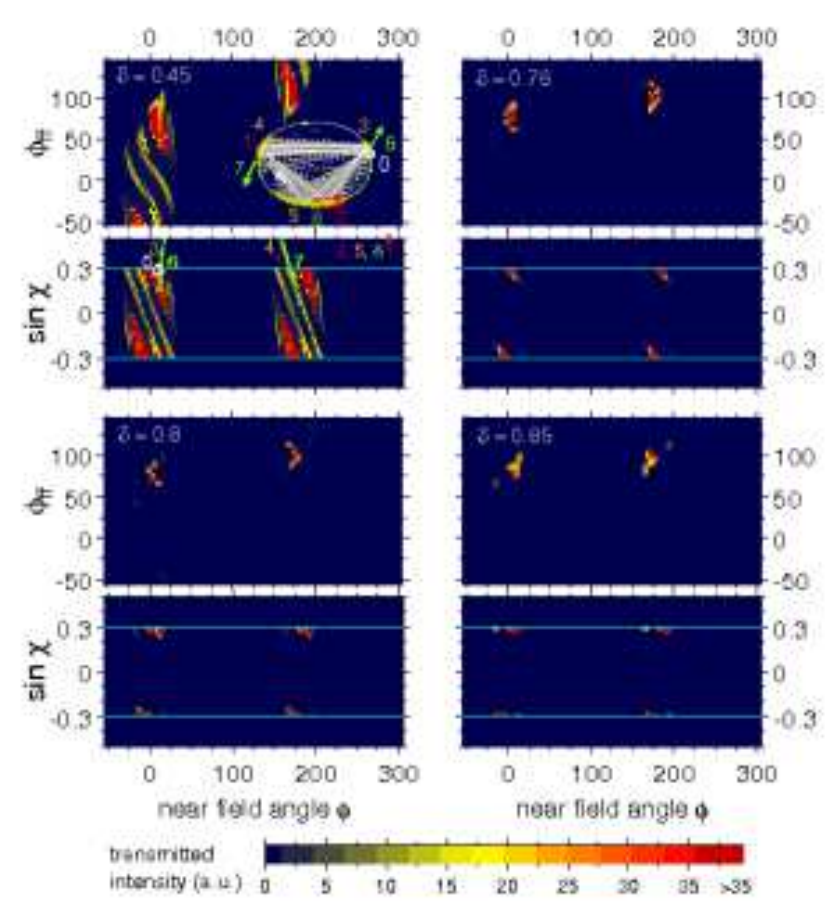

FIG. 5: (Color online) From classical phase space to the FFPs of microcavities (ray-model based simulation). The transmitted Fresnel intensity (color scale) is shown in the near field (lower panels, sine of angle of incidence $\chi$ vs. polar angle angle $\phi$ at transmission) and far field (upper panels, resulting far field angle $\phi_{\mathrm{ff}}$ vs. polar angle $\phi$ of ray origin). The region of violated total internal reflection is enclosed by light lines in the lower panels. The intensity distribution in the lower panels corresponds well to the hard-wall results (cf. the lighter points in Fig. 2); their translation into the FFP in the upper panels and in Fig. 4 is evident. The inset for $\delta=0.45$ illustrates how the details of the observed structure are related to shape-specific orbits as the one shown (see text).

son with the stadium shaped microcavity laser [9] due to dynamical eclipsing [13]: For $\delta>0$, regular (bouncing ball) islands around the stable fixed points $\sin \chi=0$ and $\phi=0^{\circ}, 180^{\circ}$ impose constraints on all other orbits. Light rays will still emerge mainly from the regions of highest curvature, but nearly tangentially, i.e., in direction of the shorter cavity axis, cf. also Figs. 2 and 5 .

In the remaining part of this Letter, we will focus on the fine structure of the FFP maxima and show how the underlying classical phase space expresses itself in this signature, cf. Figs. 4 and 5 . That the ray-model can effectively reproduce the fine structure and width of the central part of the FFP maxima becomes especially evident when looking at $\delta=0.76$ where the side peaks are clearly separated and the plateau-like structure of the central part is well described, similar arguments apply to the wide maximum with the slight dip for $\delta=0.45$. The power of our simple model is also nicely illustrated when comparing the FFPs for $\delta=0.8$ and 0.85 which are strikingly different despite the very similar cavity geometry: 
For $\delta=0.8$, a distinct minimum in the central plateau is evident in both experiment and theory which is missing for $\delta=0.85$, again in both data sets.

FFP and classical phase space - We now provide numerical evidence that these distinct differences in the fine structure of the FFP maxima originate in the structure of the underlying phase space. We already discussed the Poincaré SOS of oval (hard wall) billiards in Fig. 2 and turn now to the properties of light leaving the open system, cf. Fig. 5. The Fresnel intensity transmitted into far field direction $\phi_{\mathrm{ff}}$, originating from a certain near field angle $\phi$ and an angle of incidence $\chi$ prior to transmission, is represented in color scale histograms for the near and far field intensity (lower and upper panels, respectively). To this end the respective spaces, $(\sin \chi, \phi)$ and $\left(\phi_{\mathrm{ff}}, \phi\right)$, were divided into cells in which the Fresnel intensity of transmitted light rays was collected using the ray model described above.

Superimposing the classical phase space from Fig. 2 on the light distribution in the near field (lower panels) reveals that for all $\delta$ light rays leave indeed exclusively from the high curvature regions. Moreover, the larger $\delta$, the closer to the critical lines $|\sin \chi|=1 / n$ are the angles of incidence prior to transmission. That this is a characteristic property of the underlying classical phase space becomes even clearer when comparing the signatures in Fig. [5] with the fingerprint of light rays started at the high curvature region with near-critical incidence in the Poincaré SOS, see the lighter points in Fig. 2.

For $\delta=0.45$, the structure in the emmiting regions is richer than Fig. 2 would suggest. This is related to the intricate interplay between geometry, trajectory, and Fresnel's law and illustrated in the inset of Fig. 5. To this end 50 rays (marked by white circles and numbered 0 ) leaving the cavity in the high intensity region close to the critical line were followed for the next reflections (marked by numbers, colors and white trajectory are guides for the eye); those rays emit only little intensity at the first bounces. Intensity is again emitted refractively at the next subcritical reflections occuring 6 and 7 bounces later (arrows in inset). The Poincaré SOS signature of these bounces is marked by green squares (the other numbers complete the Poincaré SOS schematically): the emergence of the line-like structure in the near field Fresnel intensity (originating at bounce 7) becomes evident (symmetry considerations complete the line pattern). Note that trajectories started in the intensity gaps couple out refractively after few bounces but not once the steady phase space distribution is reached.

The upper panels of Fig. 5 show how the near field patterns are translated into the far fields, the correspondence is evident. Since the FFPs shown in Fig. 4 are obtained by summing the Fresnel intensities in the upper panels horizontally (over all $\phi$ ), we conclude that the observed far-field characteristics of (even) the lasing cavity is intimately connected with, and can nicely be explained by, the classical phase-space structure. Moreover, origin and evolution of the fine structure of the FFP maxima in Fig. 4 are easily understood in the representation of Fig. 5. Each FFP maxima in Fig. 4 consists of two contributions corresponding to light leaving from the two regions of highest curvature. For $0.45 \leq \delta \leq 0.8$, the two contributions are separated in $\phi_{\mathrm{ff}}$ causing a multipeakstructure of the FFPs, whereas for the only slightly larger shape parameter $\delta=0.85$ they (almost) merge, in excellent agreement with experiment.

Conclusion - We fabricated oval-shaped resonant microcavity lasers of various shapes, and lasing operation was successfully established. We showed that the emitted coherent light uniquely reflects the characteristics of the underlying classical phase space. We employed a raybased model that allows us to relate the observed peaks in the FFPs convincingly and unambiguously to the phasespace structure. In particular we find that the formation of a transversal barrier in the underlying classical phase space around $\delta \lesssim 0.76$ can be detected by coherent light.

We thank Susumu Shinohara and Satoshi Sunada for helpful discussions. M.H. thanks the group of T.H. for warm hospitality and support. The work at ATR was supported in part by the National Institute of information and Communication Technology of Japan.

* Present address: MPIPKS Dresden, Nöthnitzer Str. 38, D-01187 Dresden, Germany

[1] For a review of quantum chaos in mesoscopic systems, see e.g. H.-J. Stöckmann Quantum Chaos: An introduction, Cambridge University Press, Cambridge, 1999 and references therein.

[2] L. L. Sohn, L. P. Kouwenhoven, and G. Schön (Editors), Mesoscopic Electron Transport, Kluver Academic Publishers, 1997.

[3] A. M. Chang, H. U. Baranger, L. N. Pfeiffer, and K. W. West, Phys. Rev. Lett. 73, 2111 (1994).

[4] H.-J. Stöckmann and J. Stein, Phys. Rev. Lett. 64, 2215 (1990); H. D. Gräf et al., Phys. Rev. Lett. 69, 1296 (1992).

[5] G. Benettin and J.-M. Strelcyn, Phys. Rev. A 17, 773 (1978).

[6] M. Hènon and J. Wisdom, Physica D 8, 157 (1983).

[7] H. Makino, T. Harayama, and Y. Aizawa, Phys. Rev. E 59, 4026 (1999).

[8] This is commensurate with the free spectral range being about $1 / 100$ of the observed lasing mode line width.

[9] T. Fukushima and T. Harayama, IEEE Journal of Selected Topics in Quantum Electronics 10, 1039 (2004).

[10] H. G. L. Schwefel, N. B. Rex, H. E. Tureci, R. K. Chang, and A. D. Stone, J. Opt. Soc. Am. B 21, 923 (2004); M. Hentschel and M. Vojta, Opt. Lett. 26, 1764 (2001).

[11] We assume transverse electric (TE) polarisation of light leaving the microcavity.

[12] S.-Y. Lee, J.-W. Ryu, T.-Y. Kwon, S. Rim, and C.M. Kim, Phys. Rev. A 72, 061801(R) (2005).

[13] J. U. Nöckel and A. D. Stone, Nature 385, 45 (1997). 